\title{
Interferometric apodization of telescope apertures
}

\section{First laboratory results obtained using a Mach-Zehnder interferometer}

\author{
A. Carlotti ${ }^{1}$, G. Ricort ${ }^{1}$, C. Aime ${ }^{1}$, Y. El Azhari ${ }^{2}$, and R. Soummer ${ }^{3}$ \\ 1 Laboratoire Universitaire d'Astrophysique de Nice (LUAN), Université de Nice Sophia-Antipolis, Parc Valrose, 06108 Nice, France \\ e-mail: [Alexis.Carlotti;Gilbert.Ricort;Claude.Aime]@unice.fr \\ 2 Laboratoire de Physique des Hautes Énergies et Astrophysique, Département de Physique, Faculté des Sciences Semlalia, \\ Université Cadi Ayyad, BP 2390, Marrakech, Morocco \\ e-mail: elazhari@ucam.ac.ma \\ 3 American Museum of Natural History (AMNH), 79th Street at Central Park West, New York, NY 10024, USA \\ e-mail: rsoummer@amnh.org
}

Received 13 July 2007 / Accepted 17 September 2007

\section{ABSTRACT}

\begin{abstract}
Aims. We seek to produce apodized apertures for application in stellar coronagraphy to help in direct detections of exoplanets. We show that chromatic apodized apertures of any shape in transmission can be obtained with a specific MZI and we demonstrate this capability in two cases.

Methods. The method takes advantage of the capabilities of the MZI, in which the two outputs correspond to the addition and subtraction of the two wave amplitudes in both arms. The result is obtained by re-imaging the entrance aperture of the telescope in the arms of the MZI where two complementary phase masks $\pm \varphi(x, y)$ are set. At the two outputs of the MZI, the re-imaged apertures interfere, and their transmissions are multiplied respectively by a factor of the form $\cos [\varphi(x, y)]$ and $i \sin [\varphi(x, y)]$. They correspond to the apodized and anti-apodized complementary outputs.

Results. We present the results obtained for two types of apodization. A 1D cosine apodization for a square aperture is obtained by introducing a thin wedge-shaped air film, slightly tilting one of the mirrors of the MZI. A 2D circular symmetric apodization of the form $\cos \left[x^{2}+y^{2}\right]$ is obtained for a circular aperture using two complementary convergent and divergent lenses as phase masks. Aperture transmissions (in intensity) and corresponding point spread functions (PSFs) are given in each case and compared to the theoretical expectations.

Conclusions. We have demonstrated the capability of the MZI to produce an apodized aperture. This result is obtained with no loss of photons, considering the fact that there are two complementary outputs. Considerations are given on the wavelength dependence of this technique.
\end{abstract}

Key words. instrumentation: high angular resolution - methods: laboratory - techniques: interferometric

\section{Introduction}

We present in this paper the first results of an interferometric technique using a Mach-Zehnder interferometer (MZI) to produce two complementary images of the entrance aperture, one of which being the apodized aperture that could find applications for the detection of exoplanets as shown by Aime et al. (2001, 2002). The interest of apodized apertures for imaging exoplanets was first pointed out by Nisenson \& Papaliolios (2001) in their proposed apodized square aperture. The basic idea is to use a telescope with a variable aperture transmission to strongly reduce the diffraction wings of the point spread function (PSF). The apodization must be strong enough to compensate for the huge difference in brightness between the star and the planet (the planet may be a million to 10 billion times fainter than its star, depending on physical parameters and the wavelength of the observation). Nisenson and Papaliolios combined the effects of aperture transmission and aperture shape.

Possibilities given by apertures with a special contour (or shaped apertures) have been explored in several papers by Kasdin $(2003 a, b)$ and Vanderbei $(2003 a, b)$. The use of shaped and apodized apertures has been known in optics for a long time, and an excellent review of this subject can be found in the paper by Jacquinot and Roizen-Dossier (1964). Shaped apertures are easier to manufacture, but a signal-to-noise study by Aime (2005b) favors apodized apertures compared to shaped apertures. Although shaped apertures present an apparently better throughput, the light intensity in the image plane is uselessly spread. In their paper, Guyon et al. (2006) also note that the extra transmission of shaped apertures results in a brighter background that hurts the SNR.

Guyon (2003) proposes a technique for producing an apodized aperture without any loss of photons. For that, an anamorphosis of the entrance aperture is obtained by a combination of two aspheric mirrors, which redistributes the light intensity in the aperture. However, off-axis sources suffer from strong aberrations, which can be cancelled by a reverse procedure after the starlight suppression. Also, the fabrication of these mirrors is not easy.

The technique implemented in the present paper follows the proposal of Aime et al. (2002) to apodize rectangular apertures using a Michelson or an MZI. An advantage of an MZI is to not lose photons thanks to its two outputs. The complementary output could be used for wavefront sensing, star tracking, and controling the apodization process. Unlike Guyon's 
proposal, because of the linearity of the process, the apodized output cannot be brighter than what could be obtained with an absorbing apodizer, the complementary energy being transferred to the second output of the MZI. The first experiments using a Michelson interferometer were conducted by Soummer (2002) and Azhari et al. (2005) to test this idea. A cosine apodization was obtained by introducing a thin wedge-shaped air film by slightly tilting a mirror.

Apodized apertures find very interesting applications when combined with a coronagraph, greatly improving its efficiency (Aime et al. 2002; Soummer et al. 2003; Ferrari et al. 2007). Aime et al. (2001) studied the performance of cosine apodization in coronagraphy and observed that their utilization was possible for a reasonably large spectral bandwidth. In fact cosine apodizations are well-suited to apodizing the entrance aperture of a coronagraph operating with a square aperture, because they are very close to the optimal prolate spheroidal functions (Slepian et al. 1961, 1971).

Among the remarkable properties of prolate spheroidal functions, these apodizations enable coronagraphy to be used in cascade to improve the stellar light rejection without loss of photons after the first stage (Aime \& Soummer 2004; Soummer et al. 2007). The transmission of the entrance aperture must approximate the prolate function much better than a cosine function. Moreover, cosine apodizations cannot be used for telescopes with central obstruction or for more complex geometries, which require the use of generalized prolate functions (Soummer 2005; Soummer et al. 2007).

Pueyo et al. (2004) highlight the fact that any transmission shape can be obtained by interferometry, if deformable mirrors are used in a Michelson interferometer. This leads to prolate apodizations by interferometry, at least for a single wavelength, since the interferometric apodization depends on wavelength. This wavelength dependence of the interferometric pattern was considered a severe problem until Aime (2005a) proposed the principle of an achromatic prolate apodized coronagraph with a Lyot mask of fixed diameter and an entrance aperture with a wavelength-dependent transmission, so that the aperture transmission remains adapted to the mask size. The transmission at each wavelength must be the prolate function that corresponds to the fixed Lyot mask. To compensate for the wavelength-scaling effects of diffraction, the apodization must be stronger in the blue than in the red, and this is what an interferometric pattern produces. This was the subject of a paper by Pueyo (2006). Unfortunately, the desired variation with the wavelength is not simple, and it is necessary to use phase masks made of at least two different materials to fit the desired variation, in the same way an achromatic lens works. This possibility opens new interests for interferometric apodization.

We have not yet reached the stage where we can produce the correct wavelength-dependent apodization to realize a perfect achromatic prolate apodized Lyot coronagraph (APALC), which is due to the difficulty of realizing the correct phase masks. We present in this paper the first results obtained with an MZI for two kinds of apodization. The first is made by a thin wedge-shaped air film, which produces similar results to those obtained with a Michelson interferometer, and the second uses phase masks to produce a circular symmetry apodization. These results can be considered as a first step toward the realizing an APALC.

The paper is organized as follows. The MZI as an additionsubtraction device is presented in Sect. 2. Results obtained for the 1D and 2D apodizations are given in Sect. 3 and conclusions are given in Sect. 4.

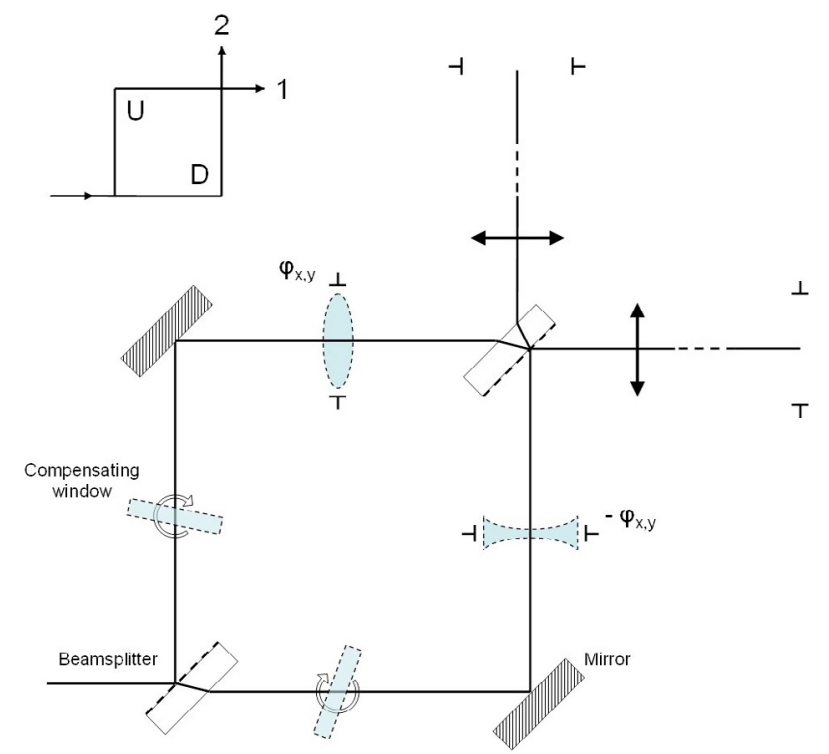

Fig. 1. The Mach-Zehnder interferometer used to produce the apodized and anti-apodized apertures. It forms a square of $0.2 \mathrm{~m}$ by $0.2 \mathrm{~m}$. An external lens forms the image of the aperture inside the MZI. For the 1D apodization, the MZI is operated without the phase masks, and the aperture image is formed on the mirrors that are slightly tilted to produce a wedge-shaped air film. For the 2D apodization, the aperture image is formed on the two complementary phase masks $\pm \varphi(x, y)$. In both cases two external lenses are used to form the two images of the aperture in the outputs $\mathrm{O} 1$ and $\mathrm{O} 2$. In the top left corner, a small drawing of the MZI displays the notation of the arms and of the outputs.

\section{The experiments}

\subsection{The $\mathrm{MZI}$ as an addition-subtraction instrument}

A schematic representation of the MZI is shown in Fig. 1. The light goes through it as indicated in the figure. The two arms of the Mach-Zehnder are denoted U (for up) and D (for down) and the outputs $\mathrm{O} 1$ and $\mathrm{O} 2$. The optical path that takes the arm $\mathrm{U}$ and is received in the output 1 is denoted U1. There are 4 possible paths (U1, U2, D1, and D2). We assume an equal optical length for all of these paths and a perfect $50 \%$ transmission and reflection of the two identical beam splitters (BS). Both U2 and D2 go across the BSs twice, U1 and D1 only once. When the MZI is used without any complementary phase term and perfectly tuned, O1 (result of U1 + D1) is bright and O2 (result of $\mathrm{U} 2+\mathrm{D} 2$ ) is dark. This well-known result can be shown easily. Denoting $\varphi_{r}$ and $\varphi_{t}$ the phases induced by the reflection and the transmission on a beam splitter, we have

O1 $: \frac{A}{2} \mathrm{e}^{\mathrm{i}\left(\varphi_{t}+\varphi_{r}\right)}+\frac{A}{2} \mathrm{e}^{\mathrm{i}\left(\varphi_{t}+\varphi_{r}\right)}=A \mathrm{e}^{i\left(\varphi_{t}+\varphi_{r}\right)}$
O2: $\frac{A}{2} \mathrm{e}^{\mathrm{i}\left(\varphi_{r}+2 \varphi_{t}\right)}+\frac{A}{2} \mathrm{e}^{\mathrm{i}\left(\varphi_{t}+\varphi_{t}\right)}=A \mathrm{e}^{i\left(2 \varphi_{t}\right)}\left(1+\mathrm{e}^{i \varphi_{r}}\right)$.

A null in the second output imposes $\varphi_{r}=\pi$. As a result, waves interact constructively in $\mathrm{O} 1$ and destructively in $\mathrm{O} 2$, and the MZI can be seen as a device producing the addition and subtraction of the complex amplitudes in both arms. The drawing of Fig. 1 corresponds to the system using two phase plates for a $2 \mathrm{D}$ apodization of the telescope aperture. For the 1D apodization, the system is simpler because the images of the aperture are simply focused on the two mirrors, with no phase masks used. 

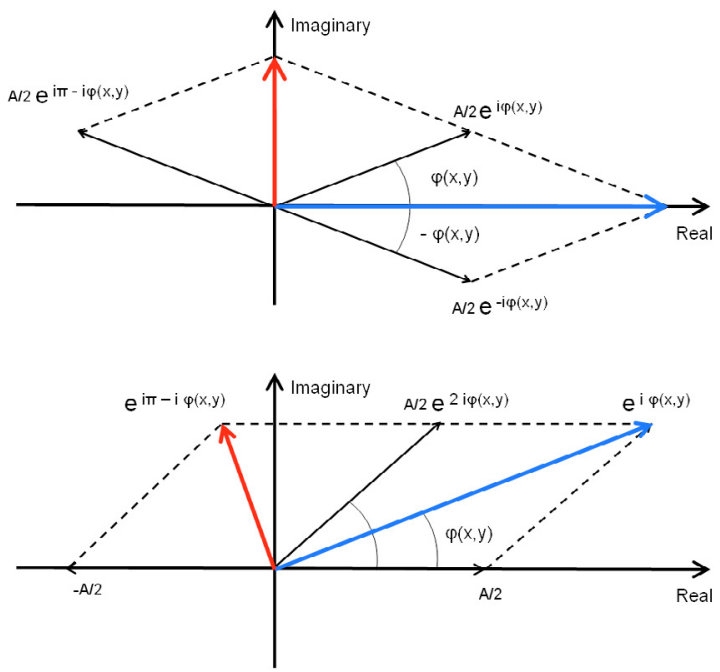

Fig. 2. Illustration of Eq. (2). Top: the apodized aperture is the result of the sum of two complex numbers, $\frac{A}{2} \mathrm{e}^{\varphi(x, y)}$ and $\frac{A}{2} \mathrm{e}^{-\varphi(x, y)}$, and the antiapodized aperture is the result of their difference. Since the amplitudes of the complex numbers are identical, the resulting sum and difference are at $90^{\circ}$; more important, they are respectively real and imaginary. Bottom: using only a single phase term in one of the arms of the MZI will give the correct amplitude for the transmissions $t_{1}(x, y)$ and $t_{2}(x, y)$, but will also produce an unwanted space-variable phase term. For the resulting vectors, the phase is indicated to highlight that the complex numbers associated to them are not only real or imaginary.

Neglecting the irrelevant phase terms, we have

$$
\begin{aligned}
& t_{1}(x, y)=\frac{A}{2} \mathrm{e}^{\mathrm{i} \varphi(x, y)}+\frac{A}{2} \mathrm{e}^{-\mathrm{i} \varphi(x, y)}=A \cos [\varphi(x, y)] \\
& t_{2}(x, y)=\frac{A}{2} \mathrm{e}^{\mathrm{i} \varphi(x, y)}-\frac{A}{2} \mathrm{e}^{-\mathrm{i} \varphi(x, y)}=\mathrm{i} A \sin [\varphi(x, y)] .
\end{aligned}
$$

The phase shift of $\frac{\pi}{2}$ (or i) is illustrated at the top of Fig. 2. In intensity, we observe $A^{2} \cos ^{2}[\varphi(x, y)]$ in the first output and in the second $A^{2} \sin ^{2}[\varphi(x, y)]$, which clearly shows the conservation of energy for each point of the initial aperture.

It would seem easy to use just a single phase plate in one arm. This could give the same shape for the apodized aperture observed in intensity, at the expense of an aberration phase term, as shown at the bottom of Fig. 2. In the particular case of 2D apodizations produced by lenses, this would have been less critical since the effect is a simple change in focal length. To produce a particular transmission $t_{1}(x, y)$, we must use phase screens $\varphi(x, y)= \pm \arccos \left[t_{1}(x, y)\right]$. This can be realized with glass windows of widths $\Delta+\mathrm{e}(x, y)$ and $\Delta^{\prime}-\mathrm{e}(x, y)$, with

$\mathrm{e}(x, y)=\frac{\lambda}{2 \pi(n-1)} \arccos \left[t_{1}(x, y)\right]$

where $n \equiv n(\lambda)$ is the index of the glass. Two plane-parallel glasses drawn in the figure are necessary to compensate for the difference in width between $\Delta$ and $\Delta^{\prime}$.

We have not yet manufactured the glass plates for the exact prolate spheroidal functions. This requires custom glass plates, whose shape is a cone with a smooth edge. This shape can be found from Eq. (3) and the prolate functions. In this experiment, we used two complementary phase screens that were easy to obtain, e.g. a converging and a diverging lens.

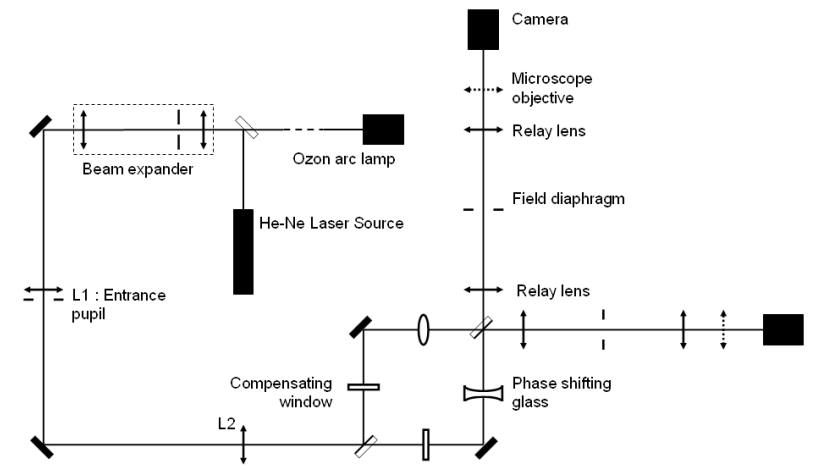

Fig. 3. The experiment. Light comes from two possible sources: a Laser or an ozone arc lamp. The lens L1 is diaphragmed by a circular or a square pupil depending on the desired apodization kind. Lens L2 reforms the image of this pupil inside the MZI. The two optical benches that follow the interferometer are identical except that one of them is observing the pupil plane and the other one the focal plane. In the latter case, a microscope objective is added before the camera.

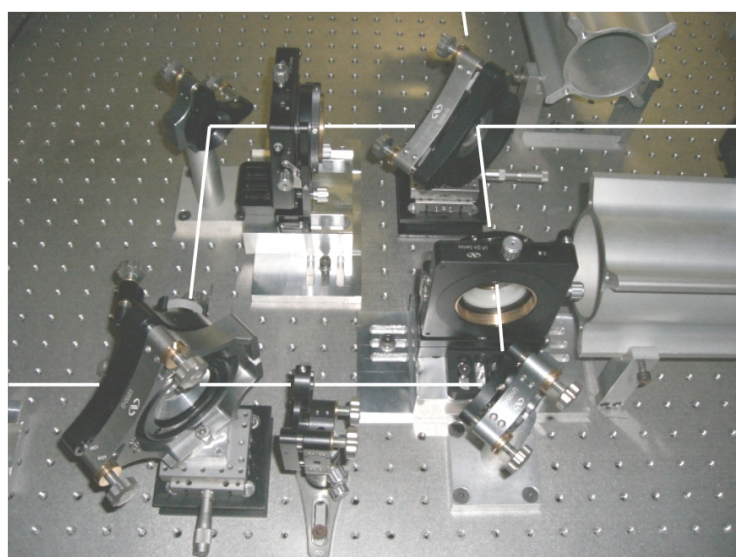

Fig. 4. Picture of the interferometer part of our experiment. Light enters the Mach-Zehnder interferometer from the left. The two compensating windows can be seen between the first beam splitter and the lateral mirrors. The mounts that contain the lenses that we use to produce our 2D apodizations are fixed before the second beamsplitter. Part of the two beams supporting imaging cameras and associated relay optics can be seen in the top right corner of the image.

\subsection{Optical setup}

The optical device we realized, and which is shown in Fig. 3, was designed to operate with both laser and white light. Here we give the results obtained in monochromatic light. Improvements are planned for the white light setup, and results will be given in a future paper. Due to space constraints, several fold mirrors were used, but we plan to remove them in the future experiment. A flip mirror injects the light of an He-Ne laser into a beam expander made of a microscope objective with focal length $16.5 \mathrm{~mm}$, a pinhole with a diameter of $5 \mu \mathrm{m}$ and a collimating lens of $200 \mathrm{~mm}$. The outgoing plane wave feeds a lens $L_{1}$ $(500 \mathrm{~mm})$, which defines the aperture of the telescope where a diaphragm is set. It may be a square or circle; the useful diameter is $8 \mathrm{~mm}$. A second lens $L_{2}(300 \mathrm{~mm})$ is used to reform a collimated beam. Lenses $L_{1}$ and $L_{2}$ are moved to form the image of the aperture either on the mirrors or on the phase plates, as previously mentioned.

We used lens of focal $200 \mathrm{~mm}$ and a 10× microscope objective to focus the apodized (or anti-apodized) aperture on a 16-bits camera. These two planes had to be observed sequentially in our 
experiment. For testing the circular symmetric apodization, we found it convenient to diaphragm the output pupil to match as closely as possible the figures of interference due to the two phase plates (here converging and diverging meniscus lenses). We used two parallel plates between the first beam splitter and the two mirrors. When rotated, they present two different optical thicknesses, and this difference is used to equalize the pathlengths in the two arms of the interferometer. The main reason for the pathlength's inequality is that the thickness of the phase masks is not equal.

The fine tuning of the Mach-Zehnder is delicate and we noticed slow drifts in the resulting pattern over a few minutes, since our setup was not in a controlled environment. The recording of the PSFs were easier than that of the apertures, the exact position for which they were apodized was more obvious, especially using the comparison between apodized and anti-apodized PSFs.

\section{Results}

\subsection{One-dimensional cosine apodization for square aperture}

This one-dimensional cosine apodization has already been studied by El Azhari (2005), Pueyo (2006), and Soummer (2002). A cosine transmission for $t_{1}(x, y)$ can be obtained by tilting the mirrors of the interferometer. In the study of Aime et al. (2002), it was shown that the prolate function was best-fitted when the value of the transmission on the pupil edge was not zero. In the present study, we consider the angle between the mirrors that makes the transmission of the aperture just zero at the edges.

Results obtained for the apodized and anti-apodized apertures and the PSFs are shown in Fig. 5, and are then compared with the clear aperture case. PSFs clearly show the effect of apodization and anti-apodization in the vertical direction. The horizontal direction is unaffected by the $1 \mathrm{D}$ apodization and remains that of the clear aperture. The apodized PSF clearly shows the reduced diffraction rings, just by comparing the number of visible fringes in both directions. One can also notice the change in the size of the diffraction structures that are broader in the apodized PSF than in the unapodized one and narrower in the anti-apodized.

The reader will note the surprising behavior of the anti apodized PSF. This is due to the sine transmission of the aperture that makes the central point of the PSF equal to zero.

Analytical expressions can be found for all these amplitudes in the aperture plane and the focal plane. Denoting $L$ the side length of the square aperture, the transmission $t(x, y)$ and focal plane complex amplitude $A(\alpha, \beta)$ for the clear aperture are

$$
\begin{aligned}
t(x, y) & =\Pi\left(\frac{x}{L}\right) \Pi\left(\frac{y}{L}\right) \\
A(\alpha, \beta) & =\frac{\sin (\pi L \alpha / \lambda)}{\pi \alpha / \lambda} \frac{\sin (\pi L \beta / \lambda)}{\pi \beta / \lambda},
\end{aligned}
$$

where we used the angular positions $\alpha=x / f$ and $\beta=$ $y / f, f$ being the focal length for the observing plane. For the apodized aperture, the transmission $t_{1}(x, y)$ and focal plane amplitude $A_{1}(\alpha, \beta)$ are

$$
\begin{aligned}
t_{1}(x, y) & =\cos (\pi x / L) \Pi\left(\frac{x}{L}\right) \Pi\left(\frac{y}{L}\right) \\
A_{1}(\alpha, \beta) & =\frac{2 L}{\pi} \frac{\cos [\pi L \alpha / \lambda]}{1-4 L^{2}(\alpha / \lambda)^{2}} \frac{\sin (\pi L \beta / \lambda)}{\pi \beta / \lambda},
\end{aligned}
$$

and for the anti-apodized aperture:

$$
t_{2}(x, y)=\mathrm{i} \sin (\pi x / L) \Pi\left(\frac{x}{L}\right) \Pi\left(\frac{y}{L}\right)
$$
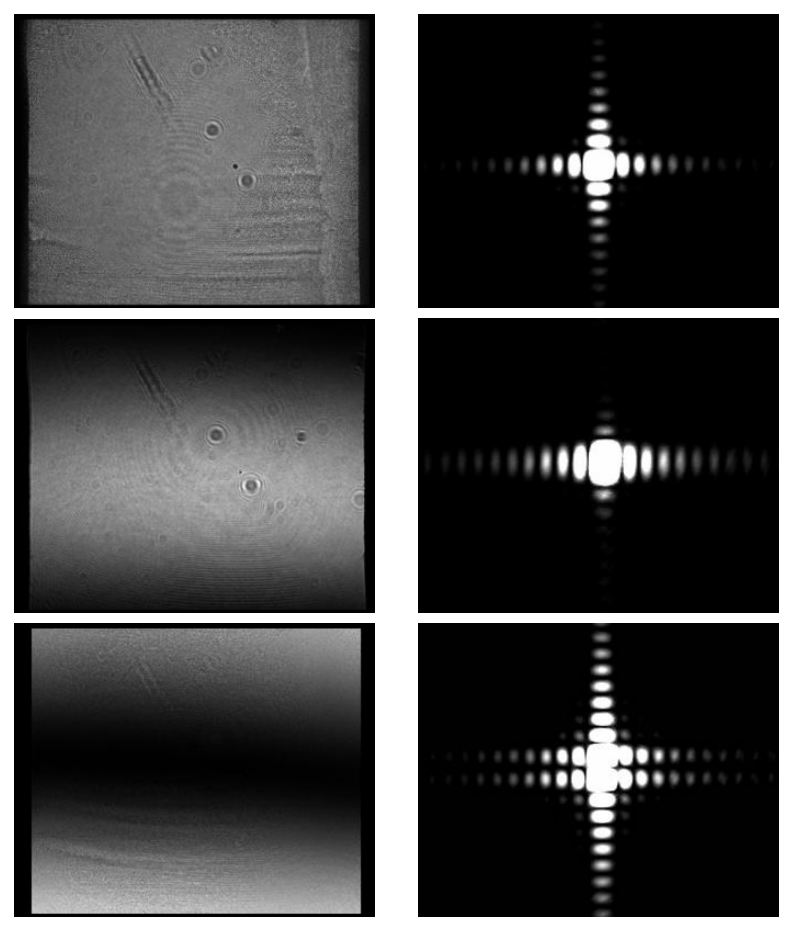

Fig. 5. One-dimension apodization. The left column shows the pupil plane and the right column the focal plane. Top: case of the unapodized pupil. Center: case of the apodized pupil. Bottom: case of the antiapodized pupil. The bright and the dark fringes are not exactly centered in the image of the pupil plane. This is due to the changes of position of the optics and of the camera between the observation of the focal plane - where the adequate adjustments were made - and the pupil plane. The maxima of all the PSFs images are the same. Intensities of the focal plane are on a log scale. Corresponding theoritical expressions are given in Eqs. (5) and (6). The $\alpha$ axis is vertical here.

$A_{2}(\alpha, \beta)=\frac{4 \mathrm{i} L^{2}}{\pi} \frac{\alpha / \lambda \cos [\pi L \alpha / \lambda]}{1-4 L^{2}(\alpha / \lambda)^{2}} \frac{\sin (\pi L \beta / \lambda)}{\pi \beta / \lambda}$

Note that the images given in Fig. 5 are intensities that should be compared to the squared modulus of the above expressions. A direct comparison between results and theory is given in Fig. 6. These results illustrate the limits of our experiment: the direct PSF or the anti-apodized PSF are well-measured, whereas the apodized PSF shows the effects of various sources of noise, since the intensity levels are much lower.

The apodization profiles show some differences with the theory, for example they are not perfectly symmetric. Simulations were conducted to test the effects of some perturbations. Analytical calculations indicate that the asymmetries in the PSFs may be due to a difference in intensity between the two arms of the MZI. Another unexpected result is that the apodized profile is higher than what is expected in theory. The pupil size may not be quite adapted to the apodization pattern, since its width is controlled by the the rotation of the lateral mirrors of the MZI. The precision of their rotation is a limit for us. It seems important here to emphasize that the study of 1D apodization was intended to give both preliminary results and information on the MZI, before tackling the $2 \mathrm{D}$ apodization presented in the next section. 

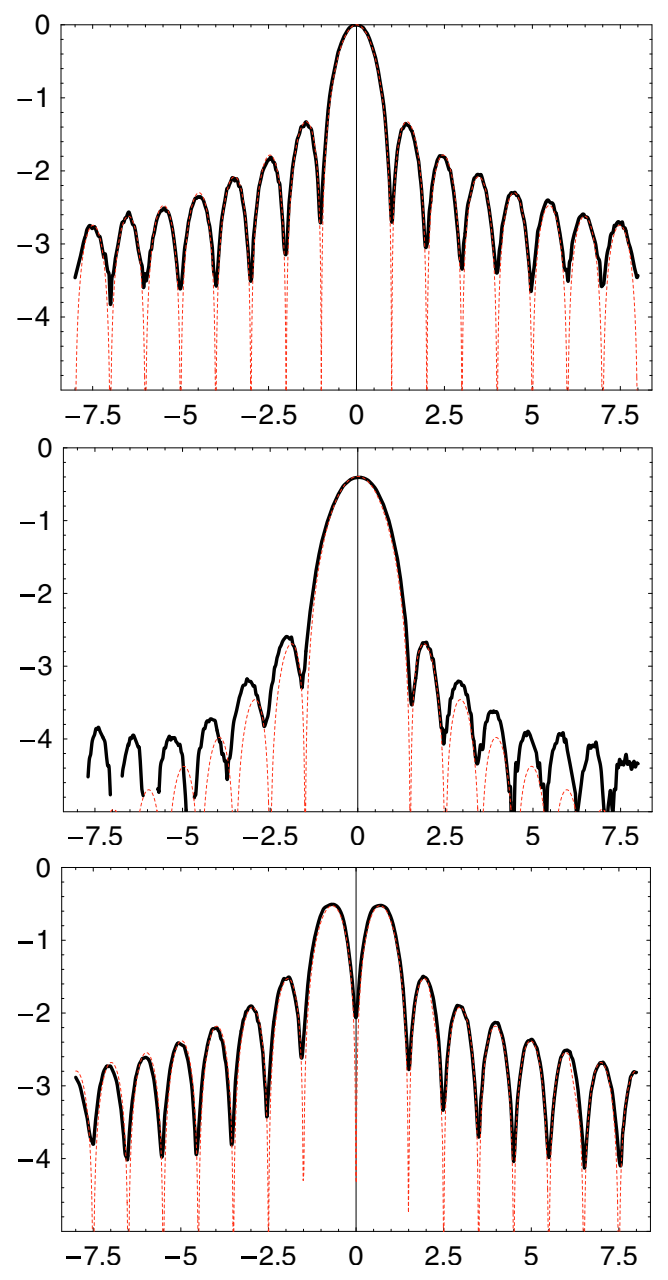

Fig. 6. Theoretical predictions and the corresponding experimental results in the case of the 1D apodization. The positions of the plots correspond to those of the images in Fig. 5. The horizontal axis is in units of $\lambda / D$ and the vertical axis is normalized intensity on a log scale. All the curves are normalized by their integrated energy. The assymmetry of the apodized PSF comes from a slight difference in the intensity transmission of the two arms, probably due to some small speck of dirt on the optical surfaces.

\subsection{Two-dimensional circular apodization}

We used two commercial lenses to produce the complementary phase plates that appear in Fig. 1. Transmissions of these lenses can be written as

$$
\Gamma_{+}(x, y)=\exp \left(-\frac{\mathrm{i} \pi\left(x^{2}+y^{2}\right)}{\lambda F}\right) ; \Gamma_{-}(x, y)=\exp \left(\frac{\mathrm{i} \pi\left(x^{2}+y^{2}\right)}{\lambda F}\right) .
$$

These phase screens are set on the images of the telescope aperture, modifying its focal length $f$. For one arm, the effective focal length is $f+F$, and $f-F$ for the other arm. In the experiment $F=8.33 \mathrm{~m}$. Considering the four waves U1, U2, D1, D2 independently, one may consider that the amplitude in the two focal planes (apodized and anti-apodized outputs) is the result of the sum and difference of these defocused waves. The expressions of the transmissions are:

$$
t(x, y)=\Pi\left(\frac{\sqrt{x^{2}+y^{2}}}{D}\right)
$$
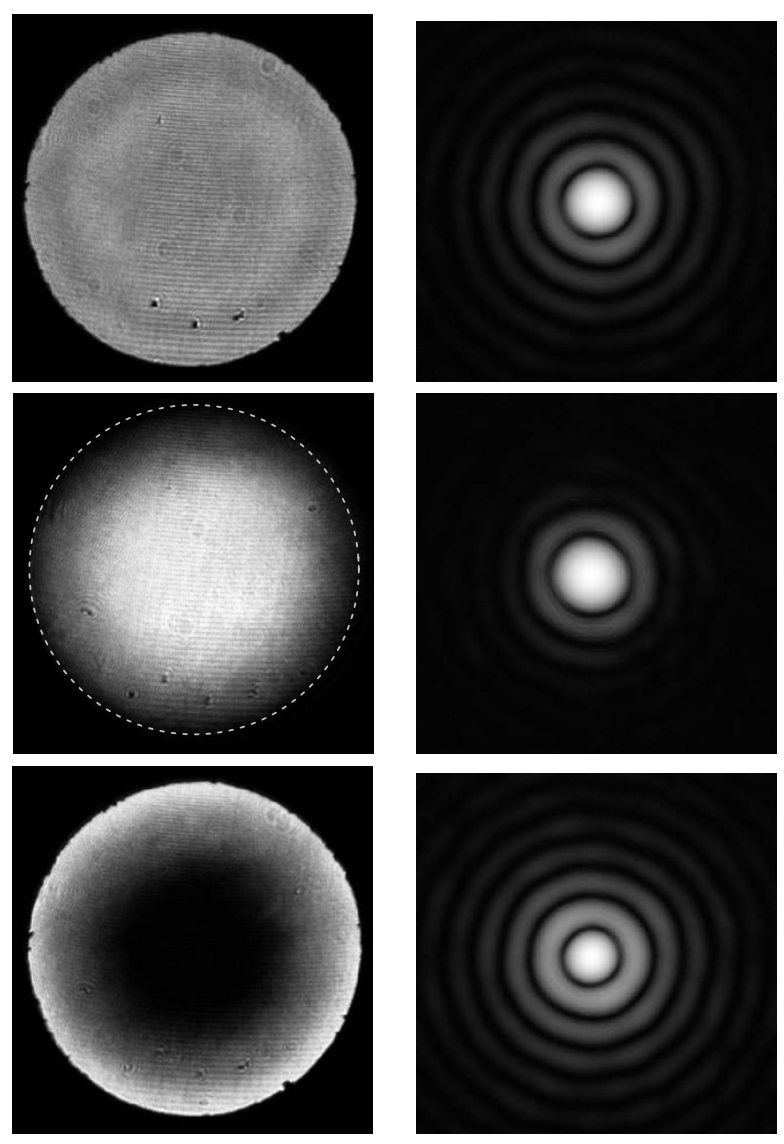

Fig. 7. Circular symetric apodization. The left column shows the pupil plane and the right column the focal plane. Top: case of the unapodized pupil. Center: case of the apodized pupil. Bottom: case of the antiapodized pupil. The dashed circle indicates the limit of the apodized pupil. The maxima of all the PSFs images are the same. Intensities of the focal plane are on a log scale.

$$
\begin{aligned}
& t_{1}(x, y)=\cos \left(\frac{\pi\left(x^{2}+y^{2}\right)}{\lambda F}\right) \Pi\left(\frac{\sqrt{x^{2}+y^{2}}}{D}\right) \\
& t_{1}(x, y)=\sin \left(\frac{\pi\left(x^{2}+y^{2}\right)}{\lambda F}\right) \Pi\left(\frac{\sqrt{x^{2}+y^{2}}}{D}\right) .
\end{aligned}
$$

The focal length $F$ and the aperture diameter $D$ are matched according to $D^{2}=2 \lambda F$, so that the apodized aperture goes to zero at the edge of the aperture. In our experiment, we had $F=20 \mathrm{~cm}$ and $D=2.1 \mathrm{~mm}$. In fact, we found it more convenient to use an initial aperture with a larger diameter and to diaphragm it to match the exact $D$ value outside the MZI, in the image of the aperture.

Images of the aperture transmissions (in intensity) made by the camera and after correction for flat field and dark current are shown in Fig. 7. Defaults of the optics are more clearly visible in the aperture plane than in the focal plane where they are somewhat smoothed by the Fourier transform operation. In the aperture plane the hardly visible high-frequency fringes are probably due to some internal reflections in our experiment and enhanced by the use of the laser's coherent light. The clear aperture presents rings that stem probably from interferences between the two surfaces of the meniscus lens. In fact, to obtain the clear aperture image, we simply block one arm of the MZI and use the second one, which explains the presence of the lens inside. In the focal plane, the defaults of the optics are visible at 

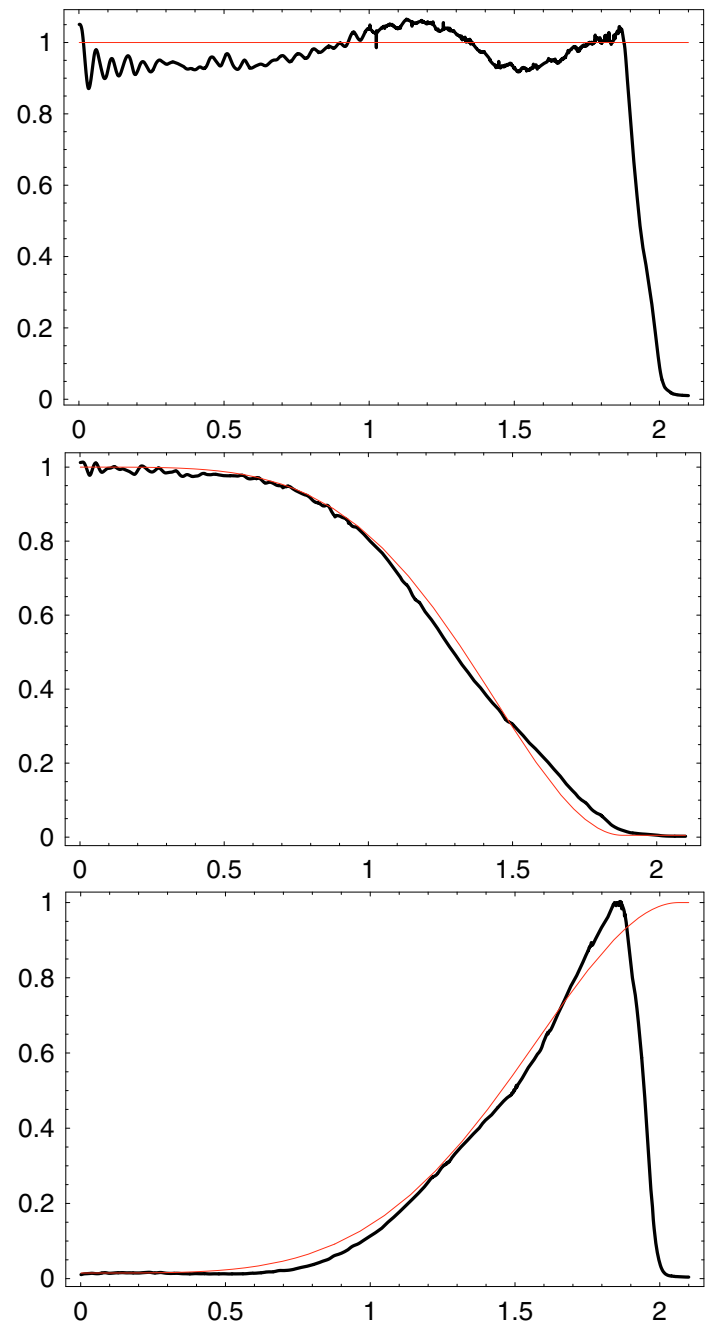

Fig. 8. Azymuthal average of the pupil intensity shown in Fig. 7 (bold curves) and the theoritical expectations drawn for comparison. The positions of the plots are as follow: at the top, case of the unapodized pupil, center: apodization case, bottom: case of the anti-apodization. The horizontal axis unit is $\mathrm{mm}$.

the origin of the break in the outer rings of the diffraction patterns.

Nevertheless, observed results agree very closely with theoretical expectations. Compared to the Airy pattern, the apodized PSF shows an enlarged central core and a significant attenuation of the rings. On the contrary, the anti-apodized PSF displays a narrower central core, at the expense of higher level of the diffraction wings, visible especially in the first ring. Figures 8 and 9 show the radial cuts of Fig. 7, on a linear scale for the aperture and on a log scale for the PSF. To improve the representation, all exprimental curves correspond to azymuthal average of the images shown in Fig. 7. In Fig. 9, the Airy pattern and the anti-apodized PSF are in very good agreement with the theoritical curves, while the apodized PSF shows more discrepencies, but for a much higher dynamic range.

\section{Conclusion}

We have built an experiment to produce an apodization of the entrance aperture of a telescope, using a Mach-Zehnder interferometer. Results already obtained with a Michelson
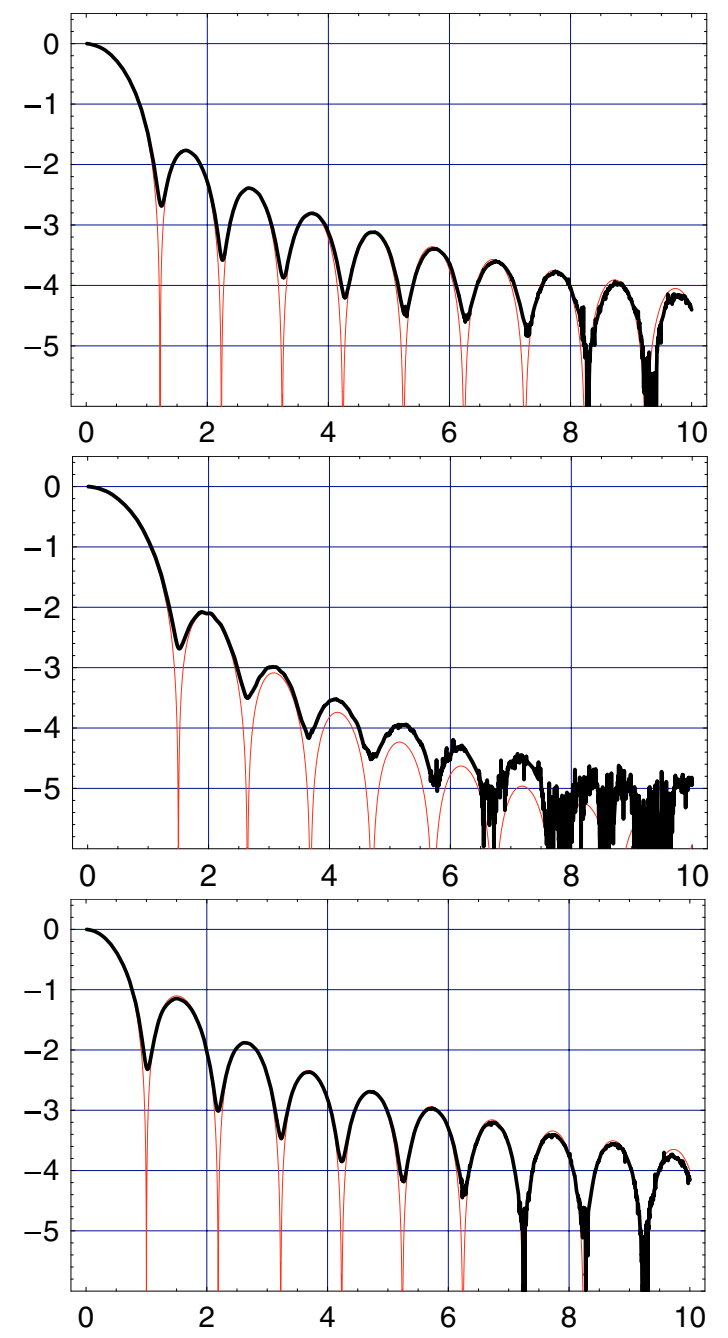

Fig. 9. Agreement between the theoritical predictions and the experimental results in the case of the circular symetric apodization. The positions of the plots correspond to those of the images in Fig. 8. The horizontal axis is in units of $\lambda / D$. The vertical axis (log scale) is normalized to the maximum of each curve.

interferometer and cited in the body of the paper for a linear apodization were confirmed and improved with the MZI. New results for circular apodizations were obtained using phase plates. Our goal was not to build an instrument ready to work on the sky but to demonstrate its apodizing capabilities. We presented our results using monochromatic light.

The MZI has two complementary outputs. When we produce an apodization in one of the outputs, an anti-apodization is created in the other. Both the pupil and focal plane of the apodized (and anti-apodized) telescope are presented in this paper. Unapodized pupils are also shown: their PSF are useful for studying the changes caused by the apodization. Our first experimental results prove that an MZI can be used effectively to produce an apodizer. The study of these apodizations has given us a better understanding of the Mach-Zehnder.

The results obtained for the 2D apodization are of better quality than in the $1 \mathrm{D}$ case. This is not intrinsic to the experiment, but simply because we improved the quality of the experimental components between the two experiments (in particular the quality of the optics). The results show, however, that improvements are still needed. We will have to include some sort of 
amplitude matching device (see the work of Weber et al. 2003), to make the intensities equal in the two arms and to compensate for the defaults of the beamsplitters. We also have to improve the tuning of the different mounts to guarantee a static and more precise apodization pattern.

Despite these experimental issues, our results demonstrate the capability of the MZI to be used as an apodizer. A more controlled environment, as well as a better optical design, is in preparation. We are working making the MZI ready to work with a polychromatic light. As prolate functions define the optimal pupil transmission, we are now studying the feasibility of a spheroidal prolate phase screen for clear and obstructed circular apertures as developped by Soummer et al. $(2005,2007)$.

\section{References}

Aime, C. 2005a, PASP, 117, 1012

Aime, C. 2005b, A\&A, 434, 785

Aime, C., \& Soummer, R. 2004, Proc SPIE, 5490, 456

Aime, C., Soummer, R., \& Ferrari, A. 2001, A\&A, 379, 697

Aime, C., Soummer, R., \& Ferrari, A. 2002, A\&A, 389, 334
El Azhari, Y., \& Al. 2005, IAU Coll., 200, 445

Ferrari, A. Soummer, R., \& Aime, C., et al. 2007, C. R. Physique, 8(3-4), 277

Frieden, B. R. 1971, Progr. Opt., 9, 311

Guyon, O. 2003, A\&A, 404, 379

Guyon, O., Pluzhnik, E. A., Kuchner, M. J., Collins, B., \& Ridgway, S. T. 2006, ApJS, 167, 81

Jacquinot, P., \& Roizen-Dossier, B. 1964, Progr. Opt., 3, 31

Kasdin, N. J., Vanderbei, R. J., Spergel, D. N., \& Littman, M. G. 2003a, Proc. SPIE, 4860, 240

Kasdin, N. J., Vanderbei, R., Spergel, D., \& Littman, M. G. 2003b, ApJ, 582, 1147

Nisenson, P., \& Papaliolios, C. 2001, ApJ, 549, L201

Pueyo, L., Give'on, A., Littman, M. G., Kasdin, N. J., \& Vanderbei, R. J. 2004, Proc. SPIE, 5490, 545

Pueyo, L., Aime, C., Soummer, R., Give'on, A., \& Kasdin, N. J. 2006, EAS Publ. Ser., 22, 239

Ridgway, S. T. 2004, EAS Publ. Ser., 12, 49

Slepian, D., \& Pollak, H. O. 1961, Bell Syst. Tech. J.

Soummer, R. 2002, PhD, Université de Nice Sophia Antipolis

Soummer, R. 2005, ApJ, 618, L161

Soummer, R., Aime, C., \& Falloon, P. 2003, A\&A, 397, 1161

Soummer, R., Pueyo, L., Ferrari, A., et al. 2007, ApJ, submitted

Vanderbei, R. J., Spergel, D. N., \& Kasdin, N. J. 2003a, ApJ, 590, 593

Vanderbei, R. J., Spergel, D. N., \& Kasdin, N. J. 2003b, ApJ, 599, 686

Weber, V., Barillot, M., Haguenauer, P., et al. 2003, EAS, 8, 319 